\title{
Long-acting muscarinic antagonist and long-acting 及2-agonist therapy to optimize chronic obstructive pulmonary disease prior to lung cancer surgery
}

\author{
TAKASHI MAKINO ${ }^{1}$, HAJIME OTSUKA ${ }^{1}$, YOSHINOBU HATA ${ }^{1}$, SATOSHI KOEZUKA ${ }^{1}$, YOKO AZUMA ${ }^{1}$, \\ KAZUTOSHI ISOBE $^{2}$, KEISHI SUGINO ${ }^{2}$, SATORU EBIHARA ${ }^{3}$, SAKAE HOMMA ${ }^{2}$ and AKIRA IYODA ${ }^{1}$ \\ ${ }^{1}$ Division of Chest Surgery, ${ }^{2}$ Division of Respiratory Medicine, ${ }^{3}$ Department of Rehabilitation Medicine, \\ Toho University School of Medicine, Ota, Tokyo 143-8541, Japan
}

Received October 24, 2017; Accepted January 17, 2018

DOI: $10.3892 / \operatorname{mco} .2018 .1595$

\begin{abstract}
Bronchodilators are essential for the perioperative management of patients with chronic obstructive pulmonary disease (COPD) undergoing surgery for lung cancer. The objective of the present study was to examine whether the usage of a long-acting $\beta 2$-agonist (LABA) with a long-acting muscarinic antagonist (LAMA) could optimize preoperative lung function and reduce the risk for postoperative pulmonary complications. Thirty-two consecutive patients with moderate-to-severe COPD who underwent a lobectomy for lung cancer and received preoperative LAMA $(n=19)$ or LAMA/LABA $(n=13)$ therapy between January 2005 and December 2015 were enrolled in this retrospective study. The improvement of preoperative pulmonary function and the postoperative morbidity were compared between the patients with preoperative LAMA, and LAMA/LABA therapy. Increases in the forced expiratory volume in one second $\left(\mathrm{FEV}_{1}\right)$ were significantly larger in the LAMA/LABA group than in the LAMA group $(0.26 \pm 0.051$ vs. $0.07 \pm 0.051 ; \mathrm{P}=0.0145$ ). More patients in the LAMA/LABA group than in the LAMA group showed a marked improvement of $>10 \%$ in $\% \mathrm{FEV}_{1}$ after bronchodilators (85 vs. $32 \%$; $\mathrm{P}=0.0046$ ). The incidence of postoperative pneumonia was significantly lower in the LAMA/LABA group than in the LAMA group ( 0 vs. 26\%; $\mathrm{P}=0.044)$. In conclusion, the present
\end{abstract}

Correspondence to: Professor Akira Iyoda, Division of Chest Surgery, Toho University School of Medicine, 6-11-1 Omori-nishi, Ota, Tokyo 143-8541, Japan

E-mail: aiyoda@med.toho-u.ac.jp

Abbreviations: COPD, chronic obstructive pulmonary disease; LAMA, long-acting muscarinic antagonist; LABA, long-acting $\beta 2$-agonist; $\mathrm{FEV}_{1}$, forced expiratory volume in one second; GOLD, Global Initiative for Chronic Obstructive Lung Disease; CT, computed tomography; SD, standard deviation; OS, overall survival

Key words: lung cancer, chronic obstructive pulmonary disease, surgery, bronchodilator, long-acting muscarinic antagonist, long-acting $\beta 2$-agonist study showed that preoperative LAMA/LABA therapy was associated with larger improvements in preoperative pulmonary function and less postoperative pneumonia than LAMA therapy. These results may lead to greater improvements in $\mathrm{FEV}_{1}$ and less postoperative pneumonia by encouraging preoperative LAMA/LABA therapy in this patient population.

\section{Introduction}

Chronic obstructive pulmonary disease (COPD) is the fourth leading cause of death and results in $>2.5$ million deaths per year worldwide (1). Lung cancer is frequently seen in patients with COPD and has been found to be the most frequent cause of death in patients with mild COPD (2). In COPD cohort studies, the incidence ratios for lung cancer ranged from 4.2 (3) to 16.7 per 1,000 person-years (4). It is crucial to improve the management of both COPD and lung cancer.

Although lung cancer should be treated appropriately according to the guidelines, lung cancer patients with COPD are at high risk when undergoing pulmonary resection because of their reduced pulmonary function and increased postoperative morbidity $(5,6)$. The perioperative management of patients with COPD and lung cancer, especially with preoperative rehabilitation and bronchodilators, is important for the improvement of surgical outcomes. Bronchodilators are one of the therapeutic options for stable COPD (7). Three types of bronchodilators are commercially available: inhaled $\beta 2$-agonists, anticholinergics, and corticosteroids. Combining bronchodilators with different mechanisms and durations of action may increase the degree of bronchodilation while producing equivalent or lesser side effects (8). Short-term combination therapy using formoterol and tiotropium has been shown to have a bigger impact on the forced expiratory volume in one second $\left(\mathrm{FEV}_{1}\right)$ than single components for COPD without lung cancer surgery $(9,10)$. Moreover, a few studies have shown that combinations of a long-acting $\beta 2$-agonist (LABA) and a long-acting muscarinic antagonist (LAMA) produced a significant increase in lung function $(11,12)$.

Previous studies reported the effectiveness of perioperative LAMA therapy in patients with COPD requiring lung cancer surgery $(13,14)$, while the effects of LAMA/LABA combination 
therapy in patients with COPD requiring lung cancer surgery are currently unclear. We hypothesized that the addition of LABA to preoperative treatment with LAMA would optimize preoperative lung function and reduce the risk for postoperative pulmonary complications. The objectives of this retrospective study were the following: i) to compare the effectiveness of perioperative LAMA/LABA therapy and LAMA therapy on lung function and the postoperative complications of patients with moderate-to-very severe COPD requiring lung cancer surgery, and ii) to examine the impact of the severity of COPD on overall survival after lung cancer surgery.

\section{Materials and methods}

Study design and population. From January 2005 to December 2015, 920 consecutive patients with primary lung cancer underwent surgical resection at our institution. Of these patients, 82 patients with moderate-to-severe COPD who required lobectomy were enrolled in a retrospective study. The severity of COPD was classified according to the Global Initiative for Chronic Obstructive Lung Disease (GOLD) criteria (7). We started preoperative LAMA/LABA therapy for all patients with COPD from 2013 and have performed this treatment for thirteen patients. Patients received LAMA therapy, as historical control according to the same inclusion and exclusion criteria, are 19 patients from 2005 to 2012. Patients meeting the following exclusion criteria were eliminated from the present analysis: No preoperative inhaled therapy $(n=25)$; a medical history of previous treatment for COPD $(n=10)$; a medical history of inhaled steroids $(n=6)$; no lung function test after inhaled therapy $(n=5)$; preoperative LAMA therapy from $2013(n=3)$ and no perioperative rehabilitation $(n=1)$. The improvement of preoperative pulmonary function and the postoperative morbidity were compared between the patients with preoperative LAMA and LAMA/LABA therapy. All patients underwent chest and abdominal computed tomography $(\mathrm{CT})$ scans, brain magnetic resonance imaging and fluoro-2-deoxyglucose positron emission tomography/CT for clinical staging for lung cancer. The following parameters were assessed from the medical records: Patient age, gender, pathological stage, histology, surgical procedure, pulmonary function, GOLD COPD stages, and prognosis. This study was reviewed and approved by the Institutional Review Board (M16021).

Smoking cessation. All patients in this study were confirmed to have ceased smoking more than 2 weeks before surgery.

Preoperative bronchodilators. In the LAMA group, the patients received inhaled tiotropium bromide $(n=19)$ from $>2$ weeks before surgery to at least 1 month after surgery without interruption.

In the LAMA/LABA group, the patients received inhaled tiotropium bromide and LABA [formoterol $(n=8)$, indacaterol $(n=4)$ ] or combined LABA/LAMA [indacaterol/glycopyrrolate $(n=1)]$ from $>2$ weeks before surgery to at least 1 month after surgery without interruption.

Postoperative complications. Postoperative pulmonary complications were defined as: i) pneumonia, defined by the presence of at least three of the following criteria: persistent lung infiltrate on chest X-ray, fever $>38.3^{\circ} \mathrm{C}$, white blood cell count $>10,000 \mathrm{~mm}^{3}$ or $<3,000 \mathrm{~mm}^{3}$; ii) acute respiratory failure, defined as postoperative ventilator dependence $>12$ h or reintubation for mechanical ventilation; iii) chronic respiratory failure, defined as the need for continuous oxygen therapy for more than 1 month after discharge. Postoperative cardiovascular complications were defined as arrhythmias (atrial fibrillation, paroxysmal supraventricular tachycardia, ventricular tachycardia), angina pectoris, myocardial infarction, congestive heart failure and thromboembolic events. Finally, surgical mortality was defined as death within 30 days following surgery.

Statistical analysis. The data are presented as the mean \pm standard deviation (SD) or as medians with interquartile ranges. Categorical variables are shown as the percentage of the sample. Comparisons between the two groups were assessed by Student's t-test for normally distributed variables or by the Mann-Whitney U test for non-normally distributed variables. Differences were considered to be statistically significant when the P-value $<0.05$. Overall survival (OS) was defined as the time from the date of surgery until the date of the last follow-up for living patients or until death. Survival curves were prepared using the Kaplan-Meier method and were compared univariately using the log-rank test. To determine which factors were significantly associated with survival, a multivariate analysis using a Cox proportional hazards model was performed. All statistical analyses were performed using JMP version 11.0 (SAS Institute Inc., Cary, NC, USA).

\section{Results}

Patient characteristics. The clinical characteristics of patients are shown in Table I. There was no significant difference between patients in the LAMA group and the LAMA/LABA group with regard to age, sex, pathological stage (AJCC 8th), Charlson index, histology, $\mathrm{SpO}_{2}, \mathrm{PaO}_{2}$, pulmonary function, and GOLD COPD stages.

Efficacy after bronchodilators. The treatment effects after LAMA therapy or LAMA/LABA therapy are shown in Table II. LAMA therapies resulted in the improvement of $\mathrm{FEV}_{1}$ (post-therapy $\mathrm{FEV}_{1} 1.41 \pm 0.09$ 1) and $\% \mathrm{FEV}_{1}$ (post-therapy $\% \mathrm{FEV}_{1} 63.7 \pm 2.95 \%$ ). LAMA/LABA therapies resulted in the improvement of $\mathrm{FEV}_{1}$ (post-therapy $\mathrm{FEV}_{1} 1.73 \pm 0.121$ ) and $\% \mathrm{FEV}_{1}$ (post-therapy $\% \mathrm{FEV}_{1}$ $78.3 \pm 3.74 \%$ ). The increases in $\mathrm{FEV}_{1}$ and $\% \mathrm{FEV}_{1}$ were significantly higher for LAMA/LABA therapy than LAMA (post-therapy-pre-therapy $\mathrm{FEV}_{1} 0.26 \pm 0.05$ vs. $0.07 \pm 0.051$, $\mathrm{P}=0.0145$; post-therapy-pre-therapy $\% \mathrm{FEV}_{1} 12.2 \pm 2.53$ vs. $4.21 \pm 2.16 \%, \mathrm{P}=0.0251$, respectively) (Fig. 1). More patients in the LAMA/LABA group had a marked improvement of $>10 \%$ in $\% \mathrm{FEV}_{1}$ after bronchodilators than patients in the LAMA group ( 85 vs. $32 \%, \mathrm{P}=0.0046$ ).

Surgical treatments. The surgical procedures performed were thoracotomy in $6(19 \%)$ and video-assisted thoracic surgery in $26(81 \%)$. Mean lengths of surgery were $306 \mathrm{~min}$, and mean perioperative blood loss was $309 \mathrm{ml}$. 
Table I. Patients characteristics.

\begin{tabular}{|c|c|c|c|}
\hline Variables & LABA + LAMA group $(n=13)$ & LAMA group $(\mathrm{n}=19)$ & P-value \\
\hline Age, years & $70.9 \pm 1.8$ & $70.8 \pm 1.5$ & 0.97 \\
\hline \multicolumn{4}{|l|}{ Sex } \\
\hline Male & $12(92 \%)$ & $17(89 \%)$ & 0.79 \\
\hline Female & $1(8 \%)$ & $2(11 \%)$ & \\
\hline Pathological stage (AJCC 8th), (I/II/III) & $7 / 3 / 3$ & $11 / 3 / 5$ & 0.87 \\
\hline Charlson index & $3.30 \pm 0.2$ & $3.47 \pm 0.1$ & 0.42 \\
\hline Histology, (Sq/Ad/LCNEC) & $7 / 5 / 1$ & $11 / 6 / 2$ & 0.91 \\
\hline $\mathrm{SpO}_{2}, \%$ & $96.2 \pm 0.3$ & $96.3 \pm 0.3$ & 0.85 \\
\hline $\mathrm{PaO}_{2}$, Torr & $82.8 \pm 2.4$ & $81.3 \pm 2.0$ & 0.64 \\
\hline \multicolumn{4}{|l|}{ Pulmonary function } \\
\hline $\mathrm{FEV}_{1} / \mathrm{FVC}, \%$ & $53.8 \pm 3.5$ & $52.3 \pm 2.5$ & 0.71 \\
\hline $\mathrm{FEV}_{1}, 1$ & $1.47 \pm 0.11$ & $1.33 \pm 0.09$ & 0.34 \\
\hline $\mathrm{FEV}_{1}, \%$ predicted & $66.1 \pm 3.26$ & $59.5 \pm 2.63$ & 0.12 \\
\hline$\%$ DLCO & $81.2 \pm 7.20$ & $79.3 \pm 5.71$ & 0.84 \\
\hline COPD-GOLD stages, (II/III) & $12 / 1$ & $15 / 4$ & 0.31 \\
\hline
\end{tabular}

Values are shown as numbers $(\%)$ or mean \pm standard deviation. LAMA, long-acting muscarinic antagonist; LABA, long-acting ß2-agonist; Sq, squamous cell carcinoma; Ad, adenocarcinoma; LCNEC, large cell neuroendocrine carcinoma; $\mathrm{SpO}_{2}$, oxygen saturation of peripheral artery; $\mathrm{PaO}_{2}$, arterial oxygen blood partial pressure; $\mathrm{FEV}_{1}$, forced expiratory ventilation in $1 \mathrm{sec}$; FVC, forced vital capacity; DLCO, diffuse capacity of the lung for carbon monoxide; COPD, chronic obstructive pulmonary disease; GOLD, Global Initiative for Obstructive Lung Disease.

Table II. Measurements after bronchodilators.

\begin{tabular}{|c|c|c|c|}
\hline Measurement & LAMA + LABA group $(n=13)$ & LAMA group $(n=19)$ & P-value \\
\hline $\mathrm{FEV}_{1}, 1$ & $1.73 \pm 0.12$ & $1.41 \pm 0.09$ & 0.0310 \\
\hline $\mathrm{FEV}_{1}, \%$ predicted & $78.3 \pm 3.74$ & $63.7 \pm 2.95$ & 0.0037 \\
\hline Increased $\mathrm{FEV}_{1}, 1$ & $0.26 \pm 0.05$ & $0.07 \pm 0.05$ & 0.0145 \\
\hline Increased $\% \mathrm{FEV}_{1}(\%$ predicted $)$ & $12.2 \pm 2.53$ & $4.21 \pm 2.16$ & 0.0251 \\
\hline \multicolumn{4}{|c|}{ Improvement of $>10 \%$ in $\mathrm{FEV}_{1}(\%$ predicted $)$} \\
\hline Yes & $11(85 \%)$ & $6(32 \%)$ & \\
\hline No & $2(15 \%)$ & $13(68 \%)$ & 0.0046 \\
\hline
\end{tabular}

Values are shown as numbers $(\%)$ or mean \pm standard deviation. LAMA, long-acting muscarinic antagonist; LABA, long-acting $\beta 2$-agonist; $\mathrm{FEV}_{1}$, forced expiratory ventilation in $1 \mathrm{sec} ; \mathrm{FVC}$, forced vital capacity.

Postoperative cardiopulmonary complications and mortality. Postoperative cardiopulmonary complications are shown in Table III. The incidence of postoperative pneumonia was significantly lower in the LAMA/LABA group than in the LAMA group ( 0 vs. $26 \%, \mathrm{P}=0.044$ ). There was no significant difference between patients in the LAMA/LABA group and the LAMA group with regard to pulmonary complications (15 vs. $42 \%, \mathrm{P}=0.109$ ), acute respiratory failure (0 vs. $11 \%$, $\mathrm{P}=0.227$ ), chronic respiratory failure ( 15 vs. $32 \%, \mathrm{P}=0.299$ ), cardiovascular complications (23 vs. $16 \%, \mathrm{P}=0.604)$, atrial fibrillation ( 15 vs. $16 \%, \mathrm{P}=0.975)$. The overall surgical mortality was $0 \%$.

Overall survival in patients with moderate-to-severe COPD requiring lung cancer surgery. The median follow-up after surgery was 20 months (range, 1 to 121). The 5-year survival rate in this study was $57 \%$. There was no significant difference between patients in the LAMA/LABA group and the LAMA group with regard to overall survival $(\mathrm{P}=0.844)$ (Fig. 2).

\section{Discussion}

The present study had two main findings. First, in this retrospective study of patients with moderate-to-severe COPD requiring lung cancer surgery, inhaled LAMA/LABA therapy was associated with greater improvements in preoperative pulmonary function than LAMA therapy. Second, LAMA/LABA therapy significantly reduced the incidence of postoperative pneumonia compared with LAMA therapy. 
Table III. The postoperative complications.

\begin{tabular}{lccr}
\hline Complications & LABA + LAMA group $(\mathrm{n}=13)(\%)$ & LAMA $(\mathrm{n}=19)(\%)$ & P-value \\
\hline Pulmonary complications & $2(15)$ & $8(42)$ & 0.109 \\
Pneumonia & $0(0)$ & $5(26)$ & 0.044 \\
Acute respiratory failure & $0(0)$ & $2(11)$ & 0.227 \\
Chronic respiratory failure & $2(15)$ & $6(32)$ & 0.299 \\
Cardiovascular complications & $3(23)$ & $3(16)$ & 0.604 \\
Atrial fibrillation & $2(15)$ & $3(16)$ & 0.975 \\
\hline
\end{tabular}

Values are shown as numbers (\%). LAMA, long-acting muscarinic antagonist; LABA, long-acting $\beta 2$-agonist.
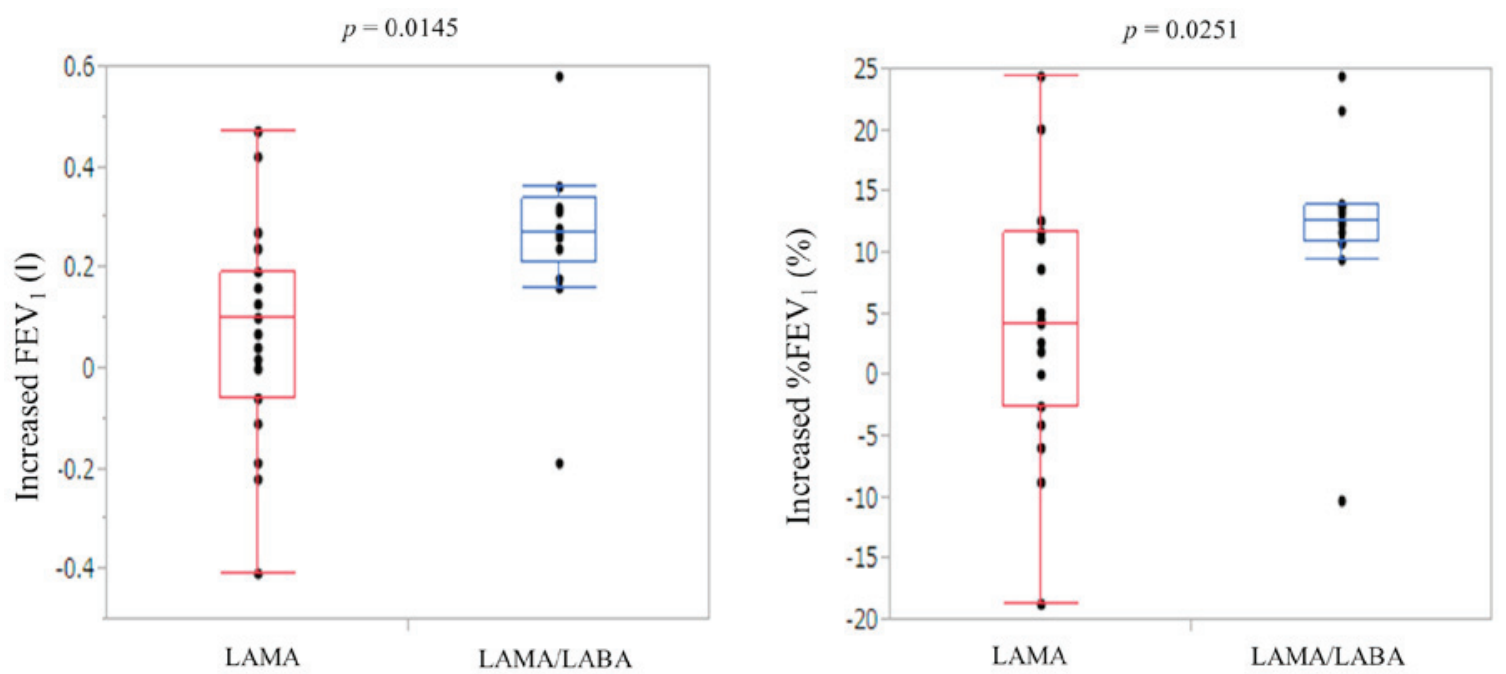

Figure 1. Significantly greater increases in $\mathrm{FEV}_{1}$ and $\% \mathrm{FEV}_{1}$ (\% predicted) in the LAMA/LABA group compared with the LAMA group. Data are presented as box-and-whisker plots. P-values were determined using Wilcoxon rank-sum tests. LAMA, long-acting muscarinic antagonist; LABA, long-acting $\beta 2$-agonist.

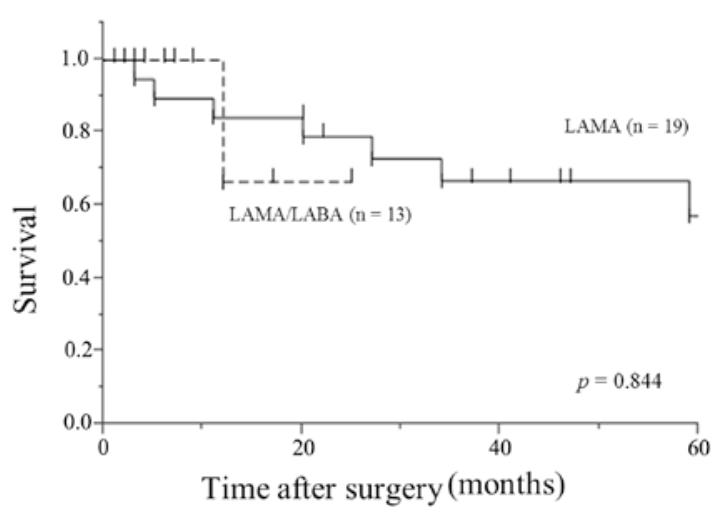

Figure 2. Five-year overall survival rates in the LAMA/LABA group and the LAMA group. Differences in overall survival between the two groups were analyzed using log-rank tests. LAMA, long-acting muscarinic antagonist; LABA, long-acting $\beta 2$-agonist.

In patients with COPD requiring lung cancer surgery, the improvement of perioperative lung function is very crucial. In a retrospective study of patients with mild-to-severe COPD requiring lung cancer surgery, 2 weeks of preoperative treatment with tiotropium significantly improved respiratory symptoms and pulmonary function as reflected by a $226-\mathrm{ml}$ increase in $\mathrm{FEV}_{1}(13,14)$. In a prospective study of patients with COPD requiring lung cancer surgery, 1 week of preoperative treatment with inhaled tiotropium/formoterol/budenoside improved pulmonary function significantly more than tiotropium/formoterol (an increase in $\mathrm{FEV}_{1}$ of $310 \mathrm{vs} .100 \mathrm{ml}$, respectively) (15). In the present study, 2-4 weeks of preoperative treatment with LAMA/LABA therapy improved pulmonary function significantly more than LAMA (an increase in $\mathrm{FEV}_{1}$ of 260 vs. $90 \mathrm{ml}$, respectively). Moreover, in $85 \%$ of the LAMA/LABA group, an improvement of $>10 \%$ in $\% \mathrm{FEV}_{1}(\%$ predicted) after treatment was achieved. These results suggest that perioperative LAMA/LABA therapy would help to achieve more curative resections in patients with COPD requiring lung cancer surgery.

COPD is an independent risk factor for morbidity and mortality in patients with lung cancer. Furthermore, patients with COPD have a fourfold increased risk of developing postoperative complications, mainly pulmonary complications $(15,16)$. Leo et al reported that up to $50 \%$ of patients with COPD develop postoperative COPD exacerbations after lung resection (17). Therefore, the perioperative management of patients with COPD and lung cancer is an important issue. Recently, several studies have reported that preoperative bronchodilators prevented postoperative complications in patients 
with COPD requiring lung cancer surgery. Bolukbas et al reported that adding budenoside to tiotropium and formoterol led to less respiratory complications ( 11 vs. $43 \%, \mathrm{P}=0.044$ ) in the postoperative period (15). Nojiri et al reported that tiotropium prevented not only respiratory complications (2 vs. $18 \%$, $\mathrm{P}=0.018$ ) but also cardiovascular complications (16 vs. $33 \%$, $\mathrm{P}=0.030$ ) in patients with COPD requiring lung cancer surgery (14) because tiotropium improved the left ventricular diastolic function in the chronic phase after pulmonary resection (18). However, Ueda et al reported that preoperative treatment with tiotropium for $\geq 7$ days improved lung function prior to thoracic surgery, but no effect on respiratory complications was seen (19). In the present study, adding LABA to LAMA significantly reduced the incidence of postoperative pneumonia compared with LAMA alone ( 0 vs. $26 \%, \mathrm{P}=0.044)$ and was not associated with more cardiovascular complications in the postoperative period ( 23 vs. $16 \%, \mathrm{P}=0.604$ ). We suspected that LAMA/LABA therapy improved the lung function and, therefore, reduced sputum retention, which accounts for the reduction in postoperative pneumonia.

This study had several limitations. Because our data were collected in a single center including a very limited number of patients for a long period and reviewed retrospectively, the study population consisted of a heterogeneous group of subjects. LABA became commercially available later than LAMA, therefore LABA/LAMA group patients distributed later than LAMA group patients. In the future, a multicenter prospective study is required for conclusion. In addition, this study is limited by the use of pre-bronchodilator spirometry. Although the diagnosis and staging of COPD should be based on post-bronchodilator spirometry as the gold standard, some guidelines recommend pre-bronchodilator spirometry because the reversibility testing is considered impractical (20).

In conclusions, our study showed that preoperative LAMA/LABA therapy was associated with greater improvements in preoperative pulmonary function than LAMA; thus, more patients with COPD who required lung cancer surgery received optimal oncological therapy. These improvements in preoperative function tended to reduce the incidence of pneumonia in the postoperative period. These results may lead to not only larger improvements in $\mathrm{FEV}_{1}$ but also less postoperative pneumonia by encouraging the addition of inhaled LABA to LAMA in this patient population. Perioperative LAMA/LABA therapy would help to achieve more curative resections and improve the surgical outcomes in patients with COPD requiring lung cancer surgery.

\section{Acknowledgements}

Not applicable.

\section{Funding}

This study was supported in part by JSPS KAKENHI [grant no. (C) JP15K10272].

\section{Availability of data and materials}

All data generated or analyzed during this study are included in this published article.

\section{Authors' contributions}

AI designed and performed the study. TM and HO analyzed all data and TM wrote the draft manuscript. YH, SK, YA, KI, KS, SE, SH and AI contributed to the acquisition of data for the present study. All authors read and approved the final manuscript.

\section{Ethics approval and consent to participate}

All participants gave individual consent to participation and for the use of all data collected during the study. This study was reviewed and approved by the Institutional Review Board (M16021).

\section{Consent for publication}

Not applicable.

\section{Competing interests}

The authors declare that they have no competing interests.

\section{References}

1. Mannino DM and Buist AS: Global burden of copd: Risk factors, prevalence, and future trends. Lancet 370: 765-773, 2007.

2. Anthonisen NR, Connett JE, Enright PL and Manfreda J: Lung Health Study Research G. Hospitalizations and mortality in the lung health study. Am J Respir Crit Care Med 166: 333-339, 2002.

3. Rabe KF, Hurd S, Anzueto A, Barnes PJ, Buist SA, Calverley P, Fukuchi Y, Jenkins C, Rodriguez-Roisin R, van Weel C, et al: Global strategy for the diagnosis, management and prevention of chronic obstructive pulmonary disease: Gold executive summary. Am J Respir Crit Care Med 176: 532-555, 2007.

4. de Torres JP, Marín JM, Casanova C, Cote C, Carrizo S, Cordoba-Lanus E, Baz-Dávila R, Zulueta JJ, Aguirre-Jaime A, Saetta M, et al: Lung cancer in patients with chronic obstructive pulmonary disease- incidence and predicting factors. Am J Respir Crit Care Med 184: 913-919, 2011.

5. Win T, Jackson A, Sharples L, Groves AM, Wells FC, Ritchie AJ and Laroche CM: Relationship between pulmonary function and lung cancer surgical outcome. Eur Respir J 25: 594-599, 2005.

6. Sekine Y, Kesler KA, Behnia M, Brooks-Brunn J, Sekine E and Brown JW: COPD may increase the incidence of refractory supraventricular arrhythmias following pulmonary resection for non-small cell lung cancer. Chest 120: 1783-1790, 2001.

7. GOLD: Global Initiative for Chronic Obstructive Lung Disease (GOLD) 2016 Global strategy for diagnosis, management and prevention of COPD. Available from http://www.goldcopd.org/.

8. Vogelmeier C, Kardos P, Harari S, Gans SJ, Stenglein S and Thirlwell J: Formoterol mono- and combination therapy with tiotropium in patients with copd: A 6-month study. Respir Med 102: 1511-1520, 2008

9. Tashkin DP, Pearle J, Iezzoni D and Varghese ST: Formoterol and tiotropium compared with tiotropium alone for treatment of COPD. COPD 6: 17-25, 2009.

10. van Noord JA, Aumann JL, Janssens E, Smeets JJ, Verhaert J, Disse B, Mueller A and Cornelissen PJ: Comparison of tiotropium once daily, formoterol twice daily and both combined once daily in patients with COPD. Eur Respir J 26: 214-222, 2005.

11. Bateman ED, Ferguson GT, Barnes N, Gallagher N, Green Y, Henley M and Banerji D: Dual bronchodilation with QVA149 versus single bronchodilator therapy: The SHINE study. Eur Respir J 42: 1484-1494, 2013.

12. Donohue JF, Maleki-Yazdi MR, Kilbride S, Mehta R, Kalberg C and Church A: Efficacy and safety of once-daily umeclidinium/vilanterol 62.5/25 mcg in COPD. Respir Med 107: 1538-1546, 2013.

13. Kobayashi S, Suzuki S, Niikawa H, Sugawara $\mathrm{T}$ and Yanai M: Preoperative use of inhaled tiotropium in lung cancer patients with untreated COPD. Respirology 14: 675-679, 2009. 
14. Nojiri T,Inoue M, YamamotoK, Maeda H,Takeuchi Y,Nakagiri T, Shintani Y, Minami M, Sawabata N, Okumura M, et al: Inhaled tiotropium to prevent postoperative cardiopulmonary complications in patients with newly diagnosed chronic obstructive pulmonary disease requiring lung cancer surgery. Surg Today 44: 285-290, 2014.

15. Bolukbas S, Eberlein M, Eckhoff J and Schirren J: Short-term effects of inhalative tiotropium/formoterol/budenoside versus tiotropium/formoterol in patients with newly diagnosed chronic obstructive pulmonary disease requiring surgery for lung cancer: A prospective randomized trial. Eur J Cardiothorac Surg 39: 995-1000, 2011.

16. Lopez-EncuentraA,AstudilloJ,CerezalJ,Gonzalez-AragonesesF, Novoa N and Sánchez-Palencia A; Bronchogenic Carcinoma Cooperative Group of the Spanish Society of Pneumology and Thoracic Surgery (GCCB-S): Prognostic value of chronic obstructive pulmonary disease in 2994 cases of lung cancer. Eur J Cardiothorac Surg 27: 8-13, 2005.
17. Leo F, Venissac N, Pop D, Solli P, Filosso P, Minniti A, Radice D, Mouroux J, Spaggiari L, Pastorino U, et al: Postoperative exacerbation of chronic obstructive pulmonary disease. Does it exist? Eur J Cardiothorac Surg 33: 424-429, 2008.

18. Nojiri T, Yamamoto K, Maeda H, Takeuchi Y, Funakoshi Y, Maekura R and Okumura M: Effects of inhaled tiotropium on left ventricular diastolic function in chronic obstructive pulmonary disease patients after pulmonary resection. Ann Thorac Cardiovasc Surg 18: 206-211, 2012.

19. Ueda K, Tanaka T, Hayashi M and Hamano K: Role of inhaled tiotropium on the perioperative outcomes of patients with lung cancer and chronic obstructive pulmonary disease. Thorac Cardiovasc Surg 58: 38-42, 2010.

20. Halpin D: Nice guidance for COPD. Thorax 59: 181-182, 2004. 